\title{
An Artificial-Intelligence Based Induction Motor Speed control and Estimation using conventional MRAS with dynamic reference modal
}

\author{
Challa Sekhar ${ }^{1}$, N.Ravisankar Reddy ${ }^{2}$ \\ ${ }^{1}$ (PG Student Department of Electrical \& Electronics Engineering, G.Pullareddy Engineering College, \\ Andhrapradesh, India \\ ${ }^{2}$ (Associate professor, Department of Electrical \& Electronics Engineering, G.Pullareddy Engineering College, \\ Andhrapradesh, India
}

\begin{abstract}
The Model Reference Adaptive System (MRAS) is probably the most widely applied speed sensor less drive control scheme. This paper proposes induction motor speed estimation using conventional MRAS and AIbased MRAS with stator resistance compensation. A conventional mathematical model based MRAS speed estimation scheme can give a relatively precise speed estimation result, but errors will occur during low frequency operation. Furthermore, it is also very sensitive to machine parameter variations. However, an AIbased MRAS system with a Stator Resistance Compensation model can improve the speed estimation accuracy and is relatively robust to parameter variations even at an extremely low frequency. These are verified by simulation results.
\end{abstract}

Keywords - Dynamic Reference Model, Model Reference Adaptive System (MRAS), Neural Networks, Induction Motor Control.

\section{Introduction}

Much effort has been devoted to speed-sensor less induction machine drive schemes, with Model Reference Adaptive System (MRAS) being the most popular [1]. In a conventional mathematical-model-based MRAS, some state variables are estimated in a reference model, (e.g. rotor flux linkage components, $\psi \mathrm{rd}, \psi \mathrm{rq}$, or back e.m.f. components, ed, eq, etc.) Of the induction machine obtained by using measured quantities, (e.g. stator currents and perhaps voltages). These reference model components are then compared with state Variables estimated using an adaptive model. The difference between these state variables is then used in an adaptation mechanism, which, for example, outputs the estimated value of the rotor speed ( $\omega r$ ) and adjusts the adaptive model until satisfactory performance is obtained [2-6]. Nevertheless, greater accuracy and robustness can be achieved, if the mathematical model is not used at all and instead, an AI-based non-linear adaptive model is employed. It is then also possible to eliminate the need of the separate PI controller, since this can be integrated into the tuning mechanism of the AI-based model [7]. However, both the conventional MRAS and AI-based MRAS scheme are easily affected by machine parameter variations, which happen during practical operation [8-9]. In this case, an online stator resistance estimator is applied to the AI-based MRAS scheme which makes the whole scheme more robust during computer simulation and could possible make the scheme usable for practical operation [10-11]. The comparison of schemes presented here is felt to be valuable since much of the literature presents results for the novel approach alone [1].

\section{Speed Estimation Using Conventional Model Reference Adaptive System}

In MRAS, there are two models, which work parallel to estimate flux-linkage of induction motor, first model name reference, and input is current and voltage, the output flux linkage of this model is to be used fiducial variable. The second model is adaptive, input of it is current and rotor speed, output flux-linkage of this model is been adjusted continuously in order that the error between those two models are turn to zero by adjusting the input of adaptive model through PI controller [12].

The expressions for the rotor flux linkages in the stationary reference frame can be obtained by using the stator voltage equations of the induction machine (in the stationary reference frame). These give (1) and (2), which are now rearranged for the rotor flux linkages:

$\psi_{r d}=\left(L_{r} / L_{m}\right)\left[\int\left(u_{s D}-R_{s} i_{s D}\right) d t-L_{s}^{\prime} i_{s D}\right] \ldots \ldots \ldots \ldots$ 
$\psi_{r q}=\left(L_{r} / L_{m}\right)\left[\int\left(u_{s Q}-R_{s} i_{s Q}\right) d t-L_{s}^{\prime} i_{s Q}\right]$

These two equations represent a so-called stator voltage model, which does not contain the rotor speed and is therefore a reference model. However, when the rotor voltage equations of the induction machine are expressed in the stationary reference frame, they contain the rotor fluxes and the speed as well. These are the equations of the adaptive model:

$$
\begin{aligned}
& \hat{\psi}_{r d}=\left(1 / T_{r}\right) \int\left(L_{m} i_{s D}-\hat{\psi}_{r d}-\hat{\omega}_{r} T_{r} \hat{\psi}_{r q}\right) d t \\
& \hat{\psi}_{r q}=\left(1 / T_{r}\right) \int\left(L_{m} i_{s Q}-\hat{\psi}_{r q}-\hat{\omega}_{r} T_{r} \hat{\psi}_{r d}\right) d t
\end{aligned}
$$

The reference and adaptive models are used to estimate the rotor flux linkages and the angular difference of the outputs of the two estimators $\varepsilon_{\omega}$ is used as the speed tuning signal.

$$
\varepsilon_{\omega}=\operatorname{Im}\left(\overline{\psi_{r}} \hat{\psi}_{r}{ }^{*} *\right)=\psi_{r q} \hat{\psi}_{r d}-\psi_{r d} \hat{\psi}_{r q}
$$

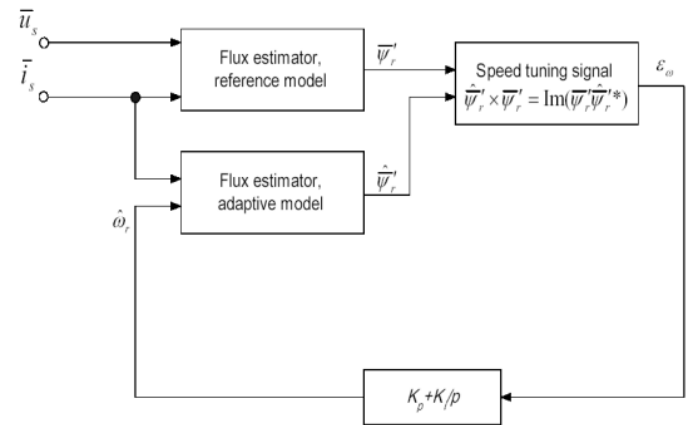

\section{Fig.1 MRAS-based rotor speed observer using rotor flux}

Linkages for the speed tuning signal

Adaptation mechanism is the PI controller to turn the error state of reference and adaptive model to zero by adjusting the input of adaptive model, which variable is the rotor speed.

By the time error state equivalent to zero the system give the estimate speed equivalent to actual speed. The estimated speed can be expressed as (5).

$\hat{\omega_{r}}=K_{p} \varepsilon_{\omega}+K_{i} \int \varepsilon_{\omega} d t$

\section{Artificial Intelligence-Based Model Reference Adaptive System}

The MRAS-based schemes described in the previous section contain a reference model and an adaptive model. However, greater accuracy and robustness can be achieved if the mathematical model is partially replaced by a neural network. It is then also possible to eliminate the need of the separate PI controller, since this can be integrated into the tuning mechanism of the neural network-based model. The neural network-based model can take various forms: it can be an artificial neural network (ANN) or a fuzzy neural network etc. and there is also the possibility of using different types of speed tuning signals. It is believed that some of these solutions can give high accuracy and are relatively robust to parameter variations even at extremely low stator frequency. One specific implementation of the ANN-based MRAS speed estimator system which is popular in academic work, as shown in Fig. 2, which is similar to the conventional MRAS system. In this new model, the adaptive model is replaced by a simple two layer neural network, which enables the whole system with fast response and better accuracy than the conventional MRAS [13-14]. 


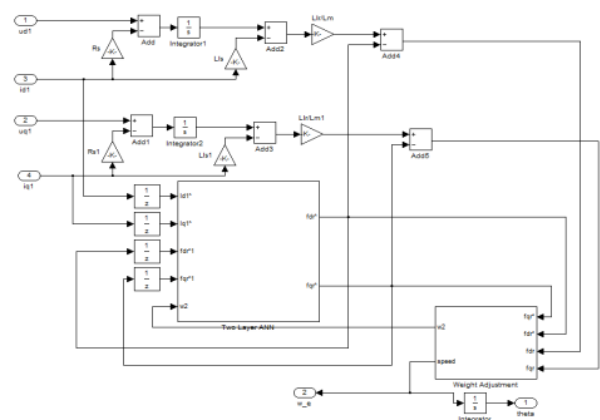

Fig.2 MRAS-based rotor speed estimator containing a two layer ANN

\section{MRAS Based Two Layer ANN Speed Estimator With Dynamic Reference Modal}

Compared to the conventional MRAS based rotor speed estimator containing a Two layer ANN could give more accurate estimation result and relatively robust to parameter variations. The two layer ANN replaces the adjustable model and adaptive mechanism in the conventional MRAS, but the reference model is still necessary for estimation the rotor flux which is used as speed tuning signal. Several machine parameters are used to build the conventional reference model, such as stator resistance $(R s)$ and stator reluctance $(L s)$. These parameters may change during the different periods of motor operating. The values of these parameters are fixed in the reference model. So the ANN speed estimator is still sensitive to parameter variations especially during the motor low speed running period. To solve this problem and make this scheme more independent to the machine parameters, a stator resistance estimator is built in the new reference model, in which the stator resistance $R s$ value could be estimated online. Fig. 3 shows the total scheme of neural network based MRAS with a dynamic reference model. In this new system, both the reference model and adaptive model of the conventional MRAS system are modified for better performance. The whole system can be divided into two main parts, the dynamic reference model part and the neural network part.

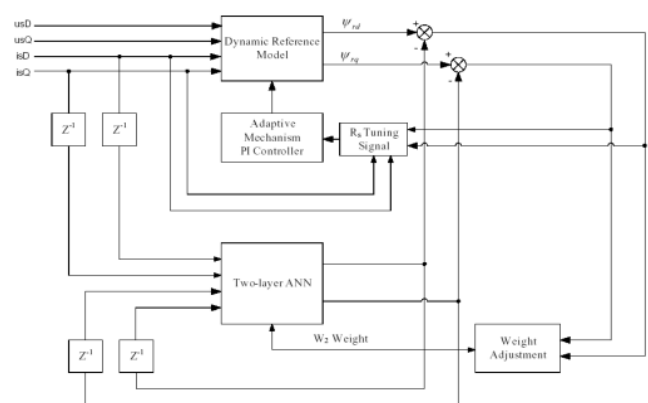

Fig.3 MRAS based ANN speed estimator with dynamic reference model.

The dynamic reference part consists of the dynamic reference model derived from equations (1) and (2), in which the stator resistance $R s$ is replaced by the online estimated value $s R^{\wedge}$ coming from equation (6) and (7),

$$
\hat{R_{s}}=\left(K_{p}+\frac{K_{i}}{p}\right) e R_{s}
$$

$e R_{s}=i_{s D}\left(\psi_{r d}-\hat{\psi}_{r d}\right)+i_{s Q}\left(\psi_{r q}-\hat{\psi_{r q}}\right)$

The neural network part contains a simple two-layer neural network, with only an input layer and an output layer. Adjustable and constant weights are built in the neural network, and the adjustable weights are proportional to the rotor speed.

The adjustable weights are changed by using the error between the outputs of the reference model and the adjustable model, since any mismatch between the actual rotor speed and the estimated rotor speed results in an error between the outputs of the reference and adaptive estimators. To obtain the required weight adjustments in the ANN, the sampled data forms of equations (3) and (4) are considered. By using the backward difference method, the sampled data forms of the equations for the rotor flux linkages can be written as (8) and (9), where $T$ is the sampling time. 
$\left[\hat{\psi}_{r d}(k)-\hat{\psi}_{r d}(k-1)\right] / T=-\hat{\psi}_{r d}(k-1) / T_{r}-\omega_{r} \hat{\psi}_{r q}(k-1)$

$+\left(L_{m} / T_{r}\right) i_{s D}(k-1)$

$\left[\hat{\psi}_{r q}(k)-\hat{\psi}_{r q}(k-1)\right] / T=-\hat{\psi}_{r q}(k-1) / T_{r}-\omega_{r} \hat{\psi}_{r d}(k-1)$

$+\left(L_{m} / T_{r}\right) i_{s Q}(k-1)$.

Thus the rotor flux linkages at the $k$ th sampling instant can be obtained from the previous $(k-1)$ th values as

$\hat{\psi_{r d}}(k)=\hat{\psi_{r d}}(k-1)\left(1-T / T_{r}\right)-\omega_{r} T \hat{\psi_{r q}}(k-1)$

$+\left(L_{m} T / T_{r}\right) i_{s D}(k-1)$

$\hat{\psi_{r q}}(k)=\hat{\psi_{r q}}(k-1)\left(1-T / T_{r}\right)-\omega_{r} T \hat{\psi_{r d}}(k-1)$

$+\left(L_{m} T / T_{r}\right) i_{s Q}(k-1)$

Introducing $c=T / T r$, the following weights are given:

$c=T / T_{r}$

$w_{1}=1-c$

$w_{2}=\omega_{r} c T_{r}=\omega_{r} T$

$w_{3}=c L_{m}$

It can be seen that $w_{1}$ and $w_{3}$ are constant weights, but $w_{2}$ is a variable weight and is proportional to the speed.

Thus Equations (10) and (11) take the following forms:

$\hat{\psi_{r d}}(k)=w_{1} \hat{\psi_{r d}}(k-1)-w_{2} \hat{\psi_{r q}}(k-1)+w_{3} i_{s D}(k-1)$

$\hat{\psi}_{r q}(k)=w_{1} \hat{\psi}_{r q}(k-1)+w_{2} \hat{\psi}_{r d}(k-1)+w_{3} i_{s Q}(k-1)$

These equations can be visualized by the very simple two layer ANN shown in Fig. 4.

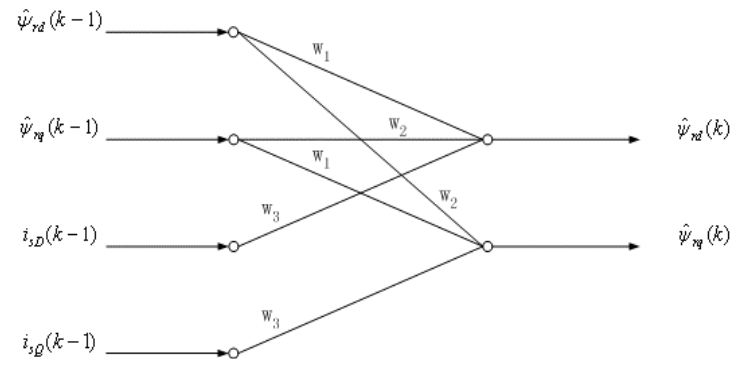

Fig.4 Neural network representation for estimated rotor flux linkages

The neural network is training by the back propagation method; the estimated rotor speed can be obtained from:

$$
\begin{aligned}
\hat{\omega}_{r}(k)=\hat{\omega}_{r}(k-1)+\Delta w_{2}(k) / T+(\alpha / T) \Delta w_{2}(k-1) & \begin{array}{l}
\hat{\omega}_{r}(k)=\hat{\omega}_{r}(k-1)+\eta \\
+(\alpha / T) \Delta w_{2}(k-1)
\end{array}
\end{aligned}
$$

Where $\eta$ is the learning rate and $\alpha$ is a positive constant called the momentum constant. The inclusion of the momentum term into the weight adjustment mechanism can significantly increase the convergence, which is 
extremely useful when the ANN shown in Fig. 4 is used to estimate in real time the speed of the induction machine.

\section{Simulation Results}

To compare the conventional MRAS and the AI-based MRAS with dynamic reference model, simulations are established by using Matlab-Simulink software, based on the standard well established validated 2-axis machine model [6]. Current, torque and speed estimation results using conventional MRAS and neural network based MRAS are shown in Fig. 5 and Fig. 6 respectively. These results assume the machine parameters are correctly measured and unchanged during operation. Both Of the two schemes can give good speed tracking results.

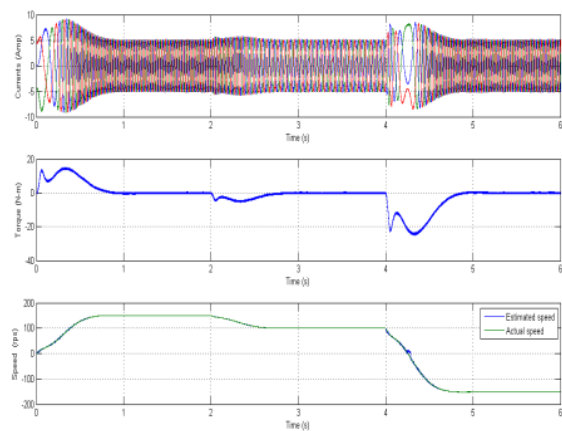

Fig. 5 Speed estimation using Conventional MRAS

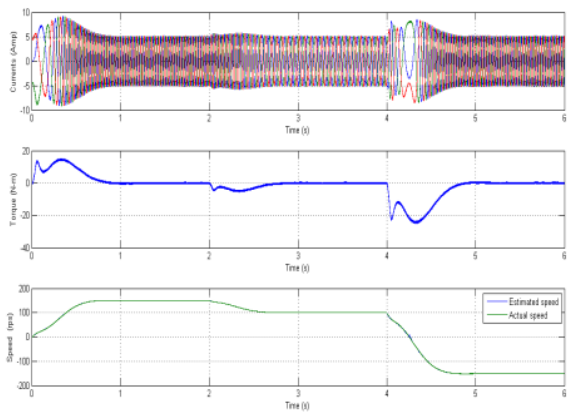

Fig. 6 Speed Estimation using Two-layer ANN MRAS

Further simulation has been carried out with changed stator resistance to test how much the parameter changing would affect the speed estimation results.

In Fig. 7 simulation is carried out with the stator resistance changed by a small amount, $2 \%$. Obviously, both schemes are still sensitive to parameter variations. A final simulation for AI-based MRAS with the dynamic reference model is shown in Fig. 9. The online estimated stator resistance is displayed in Fig.8. From the simulation result in Fig. 9, the effect caused by the stator resistance variation has been considerably improved.

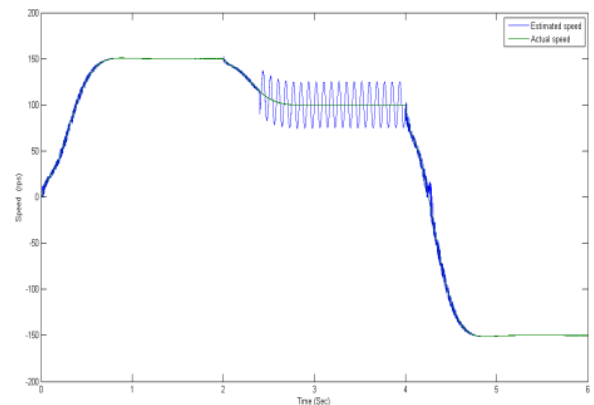

Fig.7 Speed estimation by using Conventional MRAS (with Stator resistance Rs changed 2\%) 


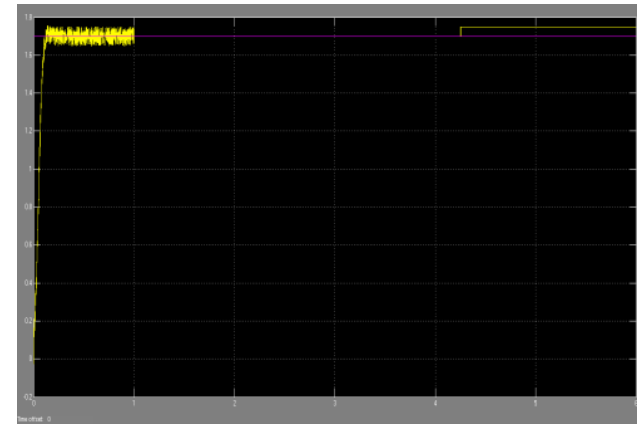

Fig. 8 Estimated Rs in the Dynamic reference model

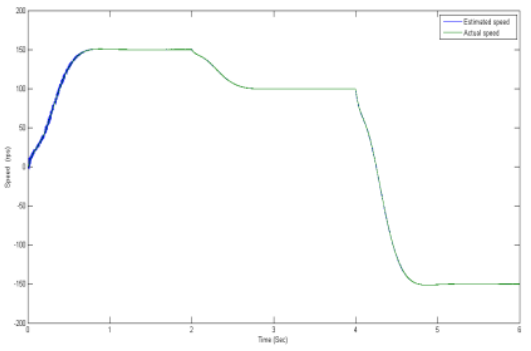

Fig. 9 Speed Estimation using Two-layer MRAS with

Dynamic reference model

Comparing all the above simulation results shows that the conventional MRAS scheme works well when the parameters are precisely measured and do not change during operation.

The MRAS with adaptive model replaced by the two-layer neural network can slightly improve the performance when working in the same situation. But both schemes can still be easily affected by parameter variations, which do occur during practical operation. By introducing the stator resistance online estimator, the performance is much improved which should enable the scheme usable for practical operation.

\section{Conclusion}

The main objective of this paper is to compare conventional MRAS and AI-based MRAS for induction motor speed sensor less speed estimation. The conventional MRAS can give good speed estimation in most of the operation period, but errors will occur during low frequency operation mainly caused by the machine parameter variations. An AI-based MRAS system can give improved accuracy and bypasses the PI controller tuning problems. The simple structure of the two-layer neural network shown in Fig. 4 yields a speed estimation system working online with a fast response.

Also the simple two-layer neural network does not require a separate learning stage, since the learning takes place during the on-line speed estimation process. This is mainly due to the fact that the development time of such an estimator is short and the estimator can be made robust to parameter variations and noise. Furthermore, in contrast to most conventional schemes, it can avoid the direct use of a speed-dependent mathematical model of the machine. However, the Two-layer neural network MRAS lies more in the realm of adaptive control then neural networks. The speed value is not obtained at the output, but as one of the weights. Moreover, only one weight is adjusted in the training. Therefore, it would still be sensitive to parameter variations and system noise. In the new approach, an online stator resistance estimator is used to compensate the parameter variations. From the comparison of the computer simulation results, it is obvious that this new approach makes the whole scheme more robust to parameter variations, which also gives the possibility of practical use of the neural network based MRAS scheme. The stator resistance estimator is working under adaptive mechanism (PI controller). Further study could be carried out for replace the PI controller with another simple neural network which could also estimate more machine parameters. 


\section{References}

[1] Finch, J. W. and Giaouris, D., Controlled AC Electrical Drives, IEEE Transactions on Industrial Electronics, Feb. 2008, 55, 1, pp. 111,

[2] Landau, Y.D., Adaptive Control the Model Reference Approach. 1979: Marcel Dekker.

[3] Vas, P., Sensor less Vector and Direct Torque Control, 1998: Oxford University Press.

[4] Shauder, C., Adaptive Speed Identification for Vector Control of Induction Motors without Rotational Transducers. IEEE Transactions on Industry Applications, 1992, 28.

[5] Yang, G. and T. Chin, Adaptive-Speed Identification Scheme for a Vector-Controlled Speed Sensor less Inverter-Induction Motors. IEEE Transactions on Industry Applications.1993,29.

[6] Fitzgerald, A.E., C. Kingsley, and S.D. Umans, Electric Machinery. $6^{\text {th }}$ ed., 2003: McGraw-Hill International Edition.

[7] Vas, P., Artificial-Intelligence-Based Electrical Machines and Drives. 1999: Oxford University Press.

[8] Kumara, I.N.S., Speed Sensor less Field Oriented Control for Induction Motor Drive. PhD Thesis, 2006, University of Newcastle upon Tyne.

[9] Leonhard, W., Controlled AC drives, a successful transition from ideas to industrial practice. Elsevier Science, 1996.

[10] Zhen, L. and L. Xu, Sensorless Field orientation Control of Induction Machines Based on Mutual MRAS scheme. IEEE Transactions on Industrial Electronics, 1998, 45.

[11] Holtz, J. and J. Quan, Drift and Parameter-Compensated Flux Estimator for Persistent Zero-Stator-Frequency Operation of Sensorless-Controlled Induction Motors. IEEE Transactions on Industrial Applications, 2003, 39.

[12] Ohtani, T., N. Takada, and K. Tanaka, Vector Control of Induction Motor without Shaft Encoder. IEEE Transactions on Industrial Applications, 1992, 28.

[13] Peng, F.Z. and T. Fukao, Robust Speed Identification for Speed Sensorless Vector Control of Induction Motors. IEEE Transactions on Industrial Applications, 1994, 30.

[14] Vasic, V. and S. Vukosavic, Robust MRAS-based Algorithm for Speed Sensorless Vector Control of Induction Motors. IEEE Power Engineering Review, 2001. 